\title{
Skull base low-grade non-intestinal adenocarcinoma in a paediatric patient*
}

Kelvin Miu, Chrysostomos Tornari, Pavol Surda

Department of Otorhinolaryngology, Guy's Hospital, Great Maze Pond, London, United Kingdom
Rhinology Online, Vol 4: $218-222,2021$

http://doi.org/10.4193/RHINOL/21.046

*Received for publication:

October 12, 2021

Accepted: November 6, 2021

Published: December 4, 2021

\begin{abstract}
Background: Non-intestinal adenocarcinomas of the sinonasal tract are uncommon neoplasms in adults, and particularly rare in the paediatric population.

Case presentation: A 10-year-old male presented to the Paediatric Otolaryngology clinic with symptoms of recurrent epistaxis, persistent clear nasal discharge, and a left-sided polypoidal swelling causing nasal obstruction. An endoscopic biopsy of the polyp under general anaesthesia found a mass arising from the anterior olfactory cleft, and the histology report described the mass as a low-grade non-intestinal adenocarcinoma. CT and MRI of the sinuses post-biopsy demonstrated no bony structure infiltration. The patient underwent a further endoscopic operation for definitive excision of the nasal mass, and the histology findings confirmed a complete resection of the tumour.
\end{abstract}

Conclusion: This case demonstrates the first case of a primary low-grade non-intestinal adenocarcinoma originating from the olfactory cleft.

Key words: Non-intestinal adenocarcinoma, paediatric, olfactory cleft, sinonasal cavity

\section{Introduction}

Sinonasal tumours present similarly in the adult and paediatric population, with unilateral symptoms of nasal blockage, anosmia, and blood-stained nasal discharge or epistaxis. Subsequent extension into adjacent structures may present with symptoms of proptosis, diplopia, epiphora, trismus, pain, or paraesthesia. Patients who present with these symptoms should be assessed with endoscopy, imaging, and biopsy ${ }^{(1,2)}$. Paediatric patients tend to present with rhabdomyosarcomas of the nasal cavity $(3,4)$, whilst adults more commonly present with squamous cell carcinomas ${ }^{(1)}$.

Primary adenocarcinomas of the sinonasal tract are a heterogeneous group of malignancies representing 10\%-20\% of all primary malignant neoplasms of the nasal cavity and paranasal sinuses in adults ${ }^{(5)}$. They can be classified as salivary or non-salivary, and further divided from non-salivary adenocarcinomas to intestinal-type adenocarcinomas (ITACs) and non-intestinal-type adenocarcinomas (non-ITACs) ${ }^{(6)}$.

Adenocarcinomas of the sinonasal cavity have previously been reported in the adult population ${ }^{(7)}$, but never for a paediatric patient. This case reports describes the first low-grade non-ITAC originating from the olfactory cleft in a child.

\section{Case presentation}

A 10-year-old male presented to the Paediatric Otolaryngology clinic with symptoms of recurrent epistaxis, persistent clear nasal discharge, and a left-sided polypoidal swelling causing nasal obstruction. An endoscopic biopsy of the polyp under general anaesthesia found a mass arising from the anterior olfactory cleft, and the histology report described the mass as a lowgrade non-intestinal adenocarcinoma. Computer Tomography (CT) and Magnetic Resonance Imaging (MRI) of the sinuses post-biopsy demonstrated no bony structure infiltration. The 

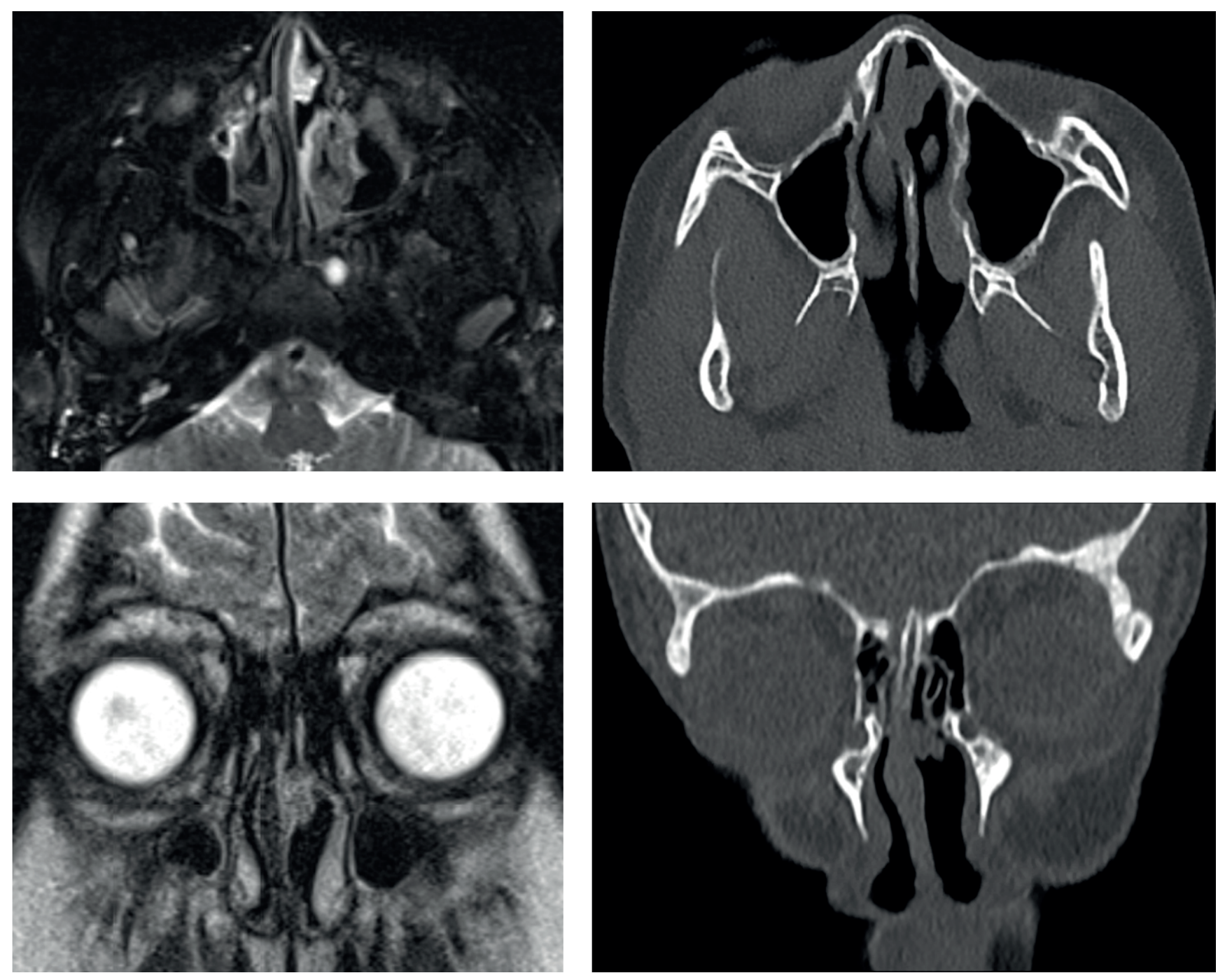

Figure 1. Axial and coronal views of T2-weighted MRI and non-contrast CT images of the left nasal mass.

patient underwent a further endoscopic operation for definitive excision of the nasal mass, and the histology findings confirmed a complete resection of the tumour.

Non-intestinal adenocarcinomas of the sinonasal tract are uncommon neoplasms in adults, and particularly rare in the paediatric population. This case demonstrates the first case of a primary low-grade non-intestinal adenocarcinoma originating from the olfactory cleft.

An endoscopic sinus surgery (ESS) biopsy of the left nasal mass was performed under general anaesthetic with ear dewaxing, dissection tonsillectomy with bipolar diathermy and suction monopolar adenoidectomy. This revealed incidental right tympanic membrane thickening and canal granulation due to infection. The left nasal mass was found to be arising from the nasal septum, medial to the middle turbinate, and adjacent to the olfactory mucosa. A limited biopsy was taken as the atypical appearance of the lump suggested a neoplasm and it is known that incomplete resection of nasal tumours leads to worse outcomes $^{(1)}$.

The specimen was submitted for histology, and was described as polypoid mucosa covered by respiratory epithelium, infiltrated by an unencapsulated neoplasm with a lobular architecture forming tubular ductal glands. The glands were arranged in a 'back-to-back' pattern, with minimal intervening stroma in the majority of the tumour. The tumour cells were uniformly roundto-ovoid, with minimal pleomorphism. There was no evidence of communication of the tumour with the surface epithelium and no surface invagination.

Alcian blue-periodic acid-Schiff (AB-PAS) highlighted the secretory component. Tumour cells were strongly and diffusely positive for Cytokeratin (CK) AE1/AE3, and CK7, variably positive for CK20 and S100, and had patchy positivity for CK 5/6. Chromo- 


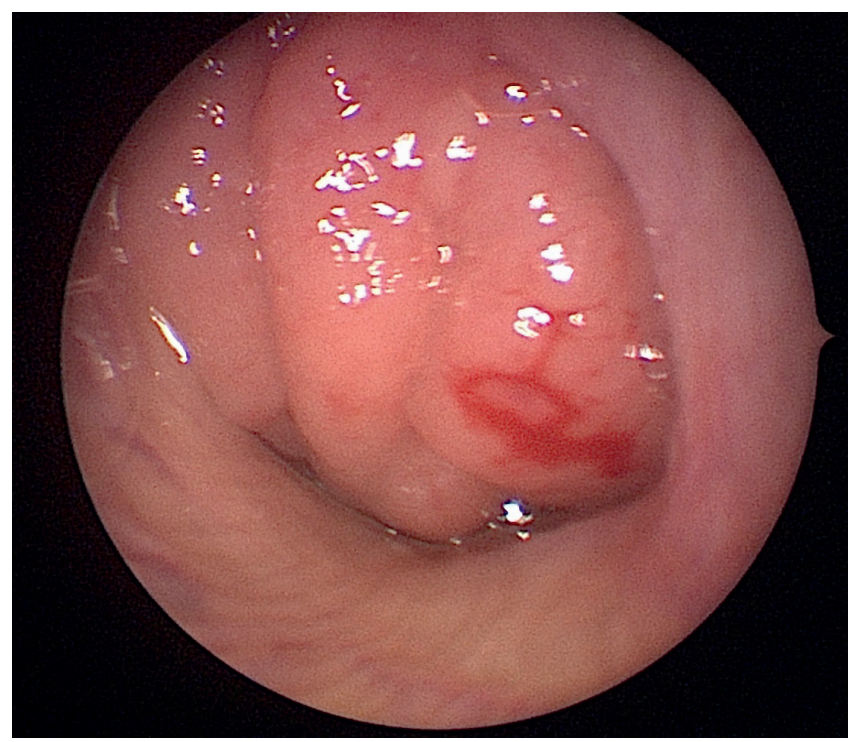

Figure 2. Endoscopic view of the left nasal mass arising from the superior aspect of the nasal cavity.

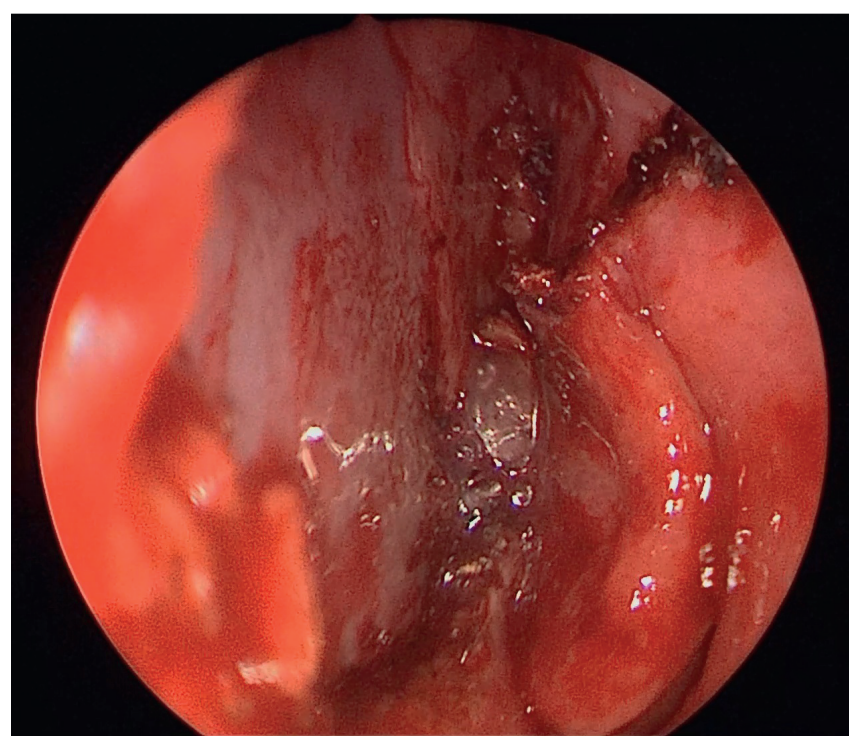

Figure 3. Endoscopic view of the inferior aspect of the left nasal cavity post-resection. granin, synaptophysin and neural cell adhesion molecule (CD56) were negative. The MiB-1 proliferation index was approximately $5 \%$. A low-grade non-intestinal sinonasal adenocarcinoma was the most likely diagnosis due to the 'back-to-back' arrangement, and staining profile - particularly the focal CK20 positivity.

This case was discussed in the local (Head \& Neck) and regional paediatric tumour Multidisciplinary Team Meeting and surgical treatment was recommended. CT and MRI of the sinuses performed post-biopsy and confirmed no anterior skull base extension.

An endoscopic resection of the left nasal mass was performed one month after the biopsy. The left nasal mid-septum to olfactory fossa area was prepared with lidocaine $5 \%$ and phenylephrine $0.5 \%$. A $1 \mathrm{~cm}$ specimen was excised from the septum (respecting the underlying cartilage) across the nasal vault to the root of the middle turbinate with a Colorado Needle. The patient was discharged on the same day with saline douches and analgesia. He had an uneventful postoperative recovery and healed by secondary intention. The specimen contained positive anterior margins, indicating a re-excision.

Re-excision of the anterior margin was completed three months later along with mapping biopsies of the posterior septum and middle turbinate. The patient again had an uneventful postoperative recovery. Histology showed only granulation tissue with no evidence of residual or recurrent tumour.

A re-discussion in the MDT confirmed negative margins and, therefore, a complete resection of the tumour.
At one month after the last ESS, the patient reported an improvement in breathing at night and denied any nasal symptoms. Examination showed adequate and equal air flow from both nostrils, and no crusting or adhesions of the operation site. No adjuvant treatment was required, and there was no evidence of recurrence at the last follow-up appointment (19 months). The patient was also discharged from the allergy clinic as he did not have any severe allergies and was subsequently lost to followup.

\section{Discussion}

Adenocarcinomas of the sinonasal tract are defined by their histopathological features and immunohistochemical staining. These tumours are classified into salivary-type adenocarcinomas, and non-salivary-type adenocarcinomas. Non-salivary-type adenocarcinomas are further sub-divided into ITACs, and nonITACS ${ }^{(8)}$.

Sinonasal salivary-type adenocarcinomas arise from the seromucinous glands and surface epithelium of the nasal cavity and paranasal sinuses. They are, therefore, histologically well-defined and resemble major and minor salivary glands.

ITACs have histological and immunohistochemical features of normal intestinal mucosa, or features of intestinal neoplasms. On immunohistochemical staining, ITACs are negative for CK7, and positive for CK20, CDX2, and villin (9-11).

Non-ITACs do not exhibit histopathological features of sinonasal salivary-type adenocarcinomas, nor ITACs, and are positive on immunohistochemical staining for CK7, and negative for CK20, CDX2, and villin (9-11). Also, in contrast to ITACs and sinonasal salivary-type adenocarcinomas, non-ITACs have no reported association with occupational or environmental carcinogens ${ }^{(8)}$. 


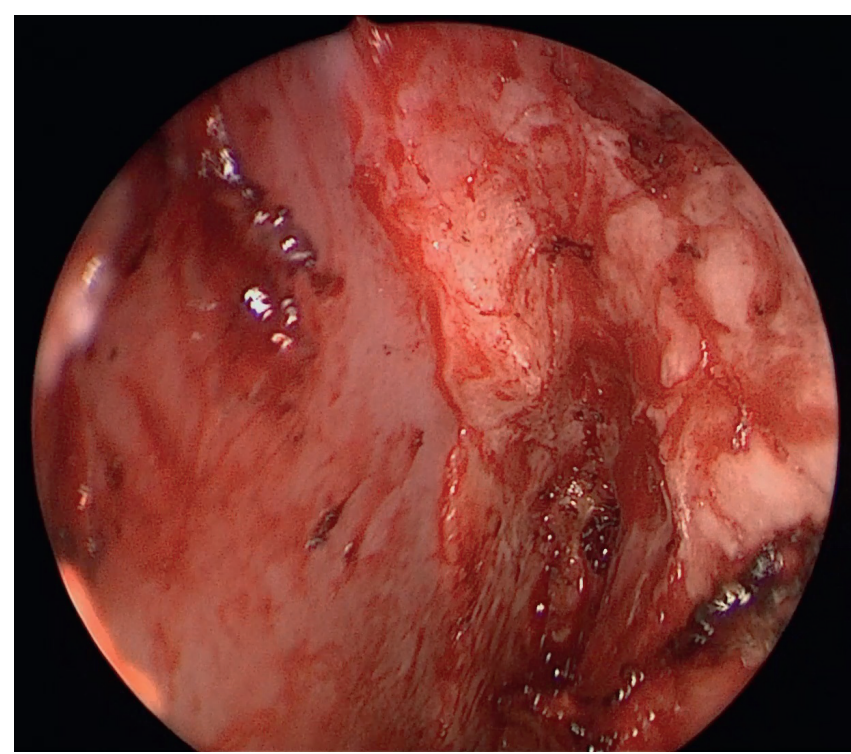

Figure 4. Endoscopic view of the superior'vault' region of the left nasal cavity post-resection.

Non-ITACs can be further divided into low-grade or high-grade types. Low-grade non-ITACs present histopathologically in an exophytic papillary and tubular, or glandular pattern. They commonly occur in the nasal cavity, ethmoid and maxillary sinus ${ }^{(8)}$, are more common in individuals above the age of 50 years, and not associated with sex or race ${ }^{(12)}$.

High-grade non-ITACS display diverse morphologic patterns such as blastomatous, apocrine, oncocytic, mucinous, poorly differentiated or undifferentiated ${ }^{(13)}$. They more frequently affect male patients with a mean age around 60 years and a wide range ${ }^{(8)}$.

Although nasal adenocarcinomas are occasionally seen in the adult population, there are no documented cases of a nasal nonITAC that present in childhood. A literature search on PubMed with key words and terms (paediatric, sinonasal, nasopharyngeal, nasopharynx, non-intestinal, adenocarcinoma) found no preceding case.

Histopathology examination of the initial specimen showed a consistent adjoining cellular arrangement and strong positive staining for $\mathrm{CK} 7$, indicative of non-ITAC, but also variably positive staining for CK20 suggestive of an ITAC. S-100 staining of the specimen was also positive though this is reported to be absent in ITACs and present to varying degrees in most non-ITAC and all sinonasal seromucinous hamartomas ${ }^{(14)}$.

The latter differential diagnosis was suggested as there is no clear criterion to differentiate this from a low-grade non-ITAC $(7,14)$. The final diagnosis favoured a low-grade non-ITAC due to the histopathological pattern, and the positive CK20 and S-100 staining results. The specimen was reviewed by two other pathologists who concurred with the diagnosis. This is there- fore the first reported paediatric case of a low grade non-ITAC of the olfactory cleft. This case demonstrates the potential of a non-ITAC to arise in the nasal cavity in paediatric patients. This should therefore be a consideration in the differential diagnosis of a sinonasal mass.

Sinonasal adenocarcinomas are described on CT as a soft tissue mass with areas of calcification representing the mucin content within the mass. CT provides detailed bony images that can identify tumour erosion through surrounding bony structures that high-grade adenocarcinomas often present with. MRI has greater contrast resolution for margins, with tumour intensity being related to mucin content, cellularity, and the presence of haemorrhage. Unilateral adenocarcinomas arising from the olfactory cleft demonstrate enlargement of the olfactory cleft and contralateral bulging of the nasal septum, whilst ethmoid sinus adenocarcinomas may present with extension to the skull base and intracranially to the frontal lobes ${ }^{(1,2)}$. In this case, staging CT and MRI was performed after the initial biopsy, and did not show any bony erosion or extension into the skull base.

The treatment standard for sinonasal low-grade non-ITACs is complete surgical removal (2), with optional postoperative radiotherapy ${ }^{(11,15)}$. Non-ITACs are usually localised and prognosis is therefore favourable in comparison to ITACs that tend to be aggressive malignancies ${ }^{(6)}$. This case was managed with an initial biopsy and complete excision of the tumour with no adjuvant treatment. Biopsy for tumour staging should deliberately leave residual tumour as an indicator to ensure a complete subsequent excision, as poorer patient outcomes occur with incomplete resection ${ }^{(1)}$. Post-operative radiotherapy was not considered as this was a low-grade tumour with no skull base extension ${ }^{(5)}$, and due to the complications of ocular and lacrimal toxicity that would have arisen ${ }^{(16,17)}$.

\section{Conclusion}

This case describes the first paediatric presentation of a low grade non-ITAC of the olfactory cleft, and demonstrates the potential of a non-ITAC to arise in the nasal cavity in paediatric patients. We therefore suggest consideration of this diagnosis in a paediatric patient who presents similarly in future.

\section{Authorship contribution}

$\mathrm{KM}$ is the main author who is responsible for conception, drafting and revision of the article. CT has contributed in the conception and revision of the article. PS has approved the final version of the article.

\section{Acknowledgments}

No acknowledgements to declare. 


\section{Funding}

None.

\section{Ethics approval and consent to participate}

Written informed consent for publication of their clinical details and clinical images has been obtained from the parent of the patient.

\section{Consent for publication}

Not applicable.

\section{Availability of data and materials}

Not applicable.

\section{Conflict of interest}

The authors declare that there are no conflicts of interest regarding the publication of this case report.

\section{References}

1. Lund V, Clarke P, Swift A, et al. Nose and paranasal sinus tumours: United Kingdom National Multidisciplinary Guidelines. J Laryngol Otol 2016; 130: 111-118.

2. Kawaguchi M, Kato $\mathrm{H}$, Tomita $\mathrm{H}$, et al Imaging Characteristics of Malignant Sinonasal Tumors. J Clin Med 2017; 6: 116.

3. Holsinger F, Hafemeister A, Hicks M, et al. Differential diagnosis of pediatric tumors of the nasal cavity and paranasal sinuses: a 45-year multi-institutional review. Ear Nose Throat J 2010; 89: 534-540.

4. Rodriguez D, Orscheln E, Koch B. Masses of the Nose, Nasal Cavity, and Nasopharynx in Children. Radiographics 2017; 37: $1704-$ 1730.

5. Leivo I. Update on Sinonasal Adenocarcinoma: Classification and Advances in Immunophenotype and Molecular Genetic Make-Up. Head Neck Pathol 2007; 1: 38-43.

6. Franchi A, Santucci M, Wenig B Adenocarcinoma. WHO histological classification of tumors of the nasal cavity and paranasal sinuses. In: Barnes L, Eveson J, Reichardt P, Sidransky D, editors. Pathology \& genetics, head and neck tumors. Lyon, France: IARC Press 2005; 1: 20-21.

7. Bullock M. Low-Grade Epithelia Proliferations of the Sinonasal Tract. Head Neck Pathol 2016; 10: 47-59.
8. Leivo I. Sinonasal Adenocarcinoma: Update on Classification, Immunophenotype and Molecular Features. Head Neck Pathol 2016; 10: 68-74

9. Abecasis J, Viana G, Pissarra C, et al. Adenocarcinomas of the nasal cavity and paranasal sinuses: a clinicopathological and immunohistochemical study of 14 cases. Histopathology 2004; 45: 254-259.

10. Franchi A, Massi D, Palomba A, et al. CDX-2, cytokeratin 7 and cytokeratin 20 immunohistochemical expression in the differential diagnosis of primary adenocarcinomas of the sinonasal tract. Virchows Arch 2004 445: 63-67.

11. Kennedy $M$, Jordan R, Berean $K$, et al. Expression pattern of CK7, CK20, CDX-2, and villin in intestinal-type sinonasal adenocarcinoma. J Clin Pathol 2004; 57: 932-937.

12. Bhaijee F, Carron J, Bell D. Low-grade nonintestinalsinonasal adenocarcinoma: a diagnosis of exclusion. Ann Diagn Pathol 2011; 15: 181-184

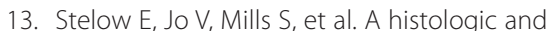
immunohistochemical study describing the diversity of tumors classified as sinonasal high-grade nonintestinal adenocarcinomas. Am J Surg Pathol 2011; 35: 971-980.

14. Purgina B, Bastaki J, Duvvuri U, et al. A Subset of Sinonasal Non-Intestinal Type Adenocarcinomas are Truly Seromucinous Adenocarcinomas: A Morphologic and
Immunophenotypic Assessment and Description of a Novel Pitfall. Head Neck Pathol 2015; 9: 436-446.

15. Heffner D, Hyams V, Hauck K, et al. Lowgrade adenocarcinoma of the nasal cavity and paranasal sinuses. Cancer 1982; 50: 312-322.

16. Shukovsky L, Fletcher G. Retinal and optic nerve complications in a high dose irradiation technique of ethmoid sinus and nasal cavity. Radiology 1972; 104: 629-634.

17. Batth S, Sreeraman R, Dienes E, et al. Clinical-dosimetric relationship between lacrimal gland dose and ocular toxicity after intensity-modulated radiotherapy for sinonasal tumours. Br J Radiol 2013; 86: 20130459 .

Dr Kelvin Miu

Department of Otorhinolaryngology

Guy's Hospital

Great Maze Pond

London, SE1 9RT

United Kingdom

E-mail: kelvin.miu@nhs.net 